\title{
Research Article \\ STUDY ON GENERATION MEAN ANALYSIS FOR SEED YIELD AND ITS ASSOCIATED TRAITS IN LINSEED (Linum usitatissimum $\mathrm{L}$.)
}

\author{
YADAV P.C. ${ }^{*}$, YADAV R.K., DUBEY S.D., SINGH H.C., VISHWANATH, PANDEY YOGESH AND KUMAR SANJEEV \\ Department of Genetics and Plant Breeding, Chandra Shekhar Azad University of Agriculture and Technology, Kanpur 208002, Uttar Pradesh, India \\ *Corresponding Author: Email-prem8250chandra@gmail.com
}

Received: February 28, 2018; Revised: March 06, 2018; Accepted: March 07, 2018; Published: March 30, 2018

\begin{abstract}
In the present study, generation mean analysis 60 treatments $\left(10 \mathrm{P}_{1}, 10 \mathrm{P}_{2}, 10 \mathrm{~F}_{1}, 10 \mathrm{~F}_{2}, 10 \mathrm{BC}_{1}\right.$ and $\left.10 \mathrm{BC}_{2}\right)$ was chosen to study the nature and magnitude of gene effects for yield and yield attributing traits. analysis of variance was carried out for all the 10 characters. Highly significant difference was observed among treatments for all the characters except days to maturity and number of primary branches per plant. Such observations were also reported. Useful heterosis over better parent and mid parent the best crosses. viz., NPHY 29 X LCK 87312, NP 121 X RR 76 and NPRR 271 X No 3 for seed yield per plant were considered. Inbreeding depression in $F_{2}$ generation was observed for all characters except number of secondary branches per plant, number of capsules per plant and seed yield per plant. Medium heritability was observed in characters like number of primary branches per plant. In $F_{1}$ generation characters likes number of seeds per capsule exhibited high value of genetic advance.
\end{abstract}

Key words- generation mean, heterosis, inbreeding depression, heritability and genetic advance

Citation: Yadav P. C., et al., (2018) Study on Generation Mean Analysis for Seed Yield and Its Associated Traits in linseed (Linum usitatissimum L.). International Journal of Microbiology Research, ISSN: 0975-5276 \& E-ISSN: 0975-9174, Volume 10, Issue 3, pp.-1027-1034. DOl: http://dx.doi.org/10.9735/0975-5276.10.3.1027-1034

Copyright: Copyright@2018 Yadav P. C., et al., This is an open-access article distributed under the terms of the Creative Commons Attribution License, which permits unrestricted use, distribution and reproduction in any medium, provided the original author and source are credited.

Academic Editor / Reviewer: Maria Toader, Vekaria Deepkumar Manojbhai

\section{Introduction}

Linseed (Linum usitatissimum L.) is a diploid ( $2 n=30$, genome size $\sim 370 \mathrm{Mb}$ ) selfpollinated annual oilseed plant. Linseed (Linum usitatissimum $L$.) is an important oilseed crop grown for both seed as well as fibre. It is mainly grown for oil purpose; it is traditionally grown for edible and industrial purposes in South East Asia (Turkmenistan, Afghanistan and India), Asia Minor and South Russia. Recent medical researchers have found linseed as best herbal source of carbohydrates, Omega -3 and Omega- 6 fatty acids, which have beneficial effect on human metabolism. The seed of linseed is a very rich source of nutrients, which contain oil $(40 \%)$, protein $(26 \%)$, fibre $(14 \%)$, mucilage $(12 \%)$, water $(9 \%)$, minerals $(4 \%)$, potassium $(0.74 \%)$, phosphorous $(0.70 \%)$, magnesium $(0.38 \%)$, calcium $(0.21 \%)$, sulphur $(0.21 \%)$, along with sodium, chlorine, iron, zinc, copper etc in traces. Lignan $(1.90-6.16 \mathrm{mg} / \mathrm{g})$ is also found in the seed. The oil contains different fatty acids like alpha linolenic acid (omega-3) $53.21 \%$, linoleic acid (omega-6) $17 \%$, oleic acid $18.51 \%$, stearic acid $4.42 \%$ and palmitic acid $4-6 \%$. Linseed is the richest source of omega-3 fatty acid and it contains almost twice as much as of omega-3 in fish oil [1-3]. The ratio of omega- 3 and omega- 6 present in linseed is about $4: 1$, so this is a best herbal source of omega-3 for improvement in human metabolism. World over, linseed is an important crop grown over 27.29 lakh ha with production of 25.20 lakh tons and average productivity of $923 \mathrm{~kg} / \mathrm{ha}$, while national production of 1.525 lakh tons is from 3.38 lakh area ha with low productivity of $473 \mathrm{~kg} / \mathrm{ha}$ [4]. As far as productivity is concerned, Indian average yield (473 kg/ha) is far below the productivity of UK (1500 kg/ha), Germany (1429 $\mathrm{kg} / \mathrm{ha}$ ), Canada (1538 kg/ha), USA (1076 kg/ha) and China (1000 kg/ha). India is an important linseed producer, which contributes about $11.82 \%$ to world acreage producing about $7 \%$ of world production.

\section{Materials and Method \\ Generation mean analysis}

(a). Building up of material: 20 parents (10 pure line female and 10 pure line male) will be crossed to develop the material. To be evaluated each female and male will be crossed only once during Rabi season 2013-14 to produce seeds of 10 straight crosses.

(b). Raising of $F_{1 s}$ material \& development of back crosses: The seeds of 10 crosses will be sown to raise $F_{1}$ population in Rabi- 2014-15. Each $F_{1}$ will be back crossed with their respective parents to produce the seeds of $B C_{1} \& B_{2}$. The parents will be maintained and fresh crosses will also be made during this season to procure $F_{1}$ seeds of straight crosses.

(c). Plan of the lay-out for experiment: All the $10 \mathrm{~F}_{1}$ 's, $10 \mathrm{~F}_{2 \mathrm{~s}}, 10 \mathrm{BC} \mathrm{C}_{1 \mathrm{~s}} \& 10 \mathrm{BC}_{2 \mathrm{~s}}$ along with their 10 pure line female \& 10 pure line male parents, will be grown in randomized block design with three replication during Rabi 2015-16 at Nawabganj Farm of CSAUA\&T Kanpur.[5-7].

Oil content (\%): Oil contain was determined by [8]. There were analysed separately for their oil content. Heterosis expressed as per-cent increase or decrease of $F_{1}$ hybrid over the best commercial check (standard heterosis) were computed as per the method $[9,10]$.

$$
\text { Heterosis over check (standard heterosis) }=\frac{\overline{F_{1}-C C}}{\overline{C C}} \times 100
$$

Where: $F_{1}=$ mean performance of $\overline{F_{1}, C C}=$ mean performance of the best commercial check.

Inbreeding depression is estimated when both $F_{1}$ and $F_{2}$ generations of the same 
cross are available. Inbreeding depression was measured as described by [10].

$$
\text { Inbreeding depression }=\frac{\overline{F_{1}}-\overline{F_{2}}}{\overline{F_{1}}} \times \mathbf{O O O}
$$

Where: $F_{1}$ and $F_{2}$ are the mean values of $F_{1}$ and $F_{2}$ progeny respectively of the same cross for a given character. Inbreeding depression may be high, medium, low and nil depending on cross.

\section{Results and Discussion \\ Analysis of variance (ANOVA)}

Analysis of variance was carried out for all the 10 characters. Treatment variance was further partitioned in orthrogenaly, it component viz. parents, $F_{1 s}, F_{2 s}$, parents vs crosses respectively. Highly significant difference was observed among treatments, for all the characters except days to maturity and number of primary branches per plant. Highly significant difference was observed among parents, for all the characters except days to $50 \%$ flowering, days to maturity, number of primary branches per plant, number of seeds per capsule, 1000-seed weight $(\mathrm{g})$ and seed yield per plant (g). Highly significant difference was observed among $F_{1} s$, for all the characters except days to maturity, number of seeds per capsule, 1000-seed weight (g) and oil content (\%). Highly significant difference observed among parent vs. crosses, for all the characters except oil content (\%). Such observation was reported by [11-13]. As regards to heterosis over better parent, significant and desirable heterosis was observed the best crosses. viz., NPHY 29 X LCK 87312, NP 121 X RR 76, NPRR 271 X No 3, RL-28-1 X L-53, AHUDERA 170 X SJKO 55 and NPHY 39 X No 3 for seed yield per plant $(g)$ were considered. It was found that increase in seed yield in almost all these crosses is due to desirable heterosis of important components traits like number of primary branches per plant, number of secondary branches per plant, number of capsules per plant, number of seeds per capsule and 1000-seed weight (g). The results of heritability coupled with genetic advance in percent of mean showed that none of the characters had moderate heritability among with high genetic advance. Rest of the traits had low to high heritability with low to moderate genetic gain in generations. Such observations were reported by [14].

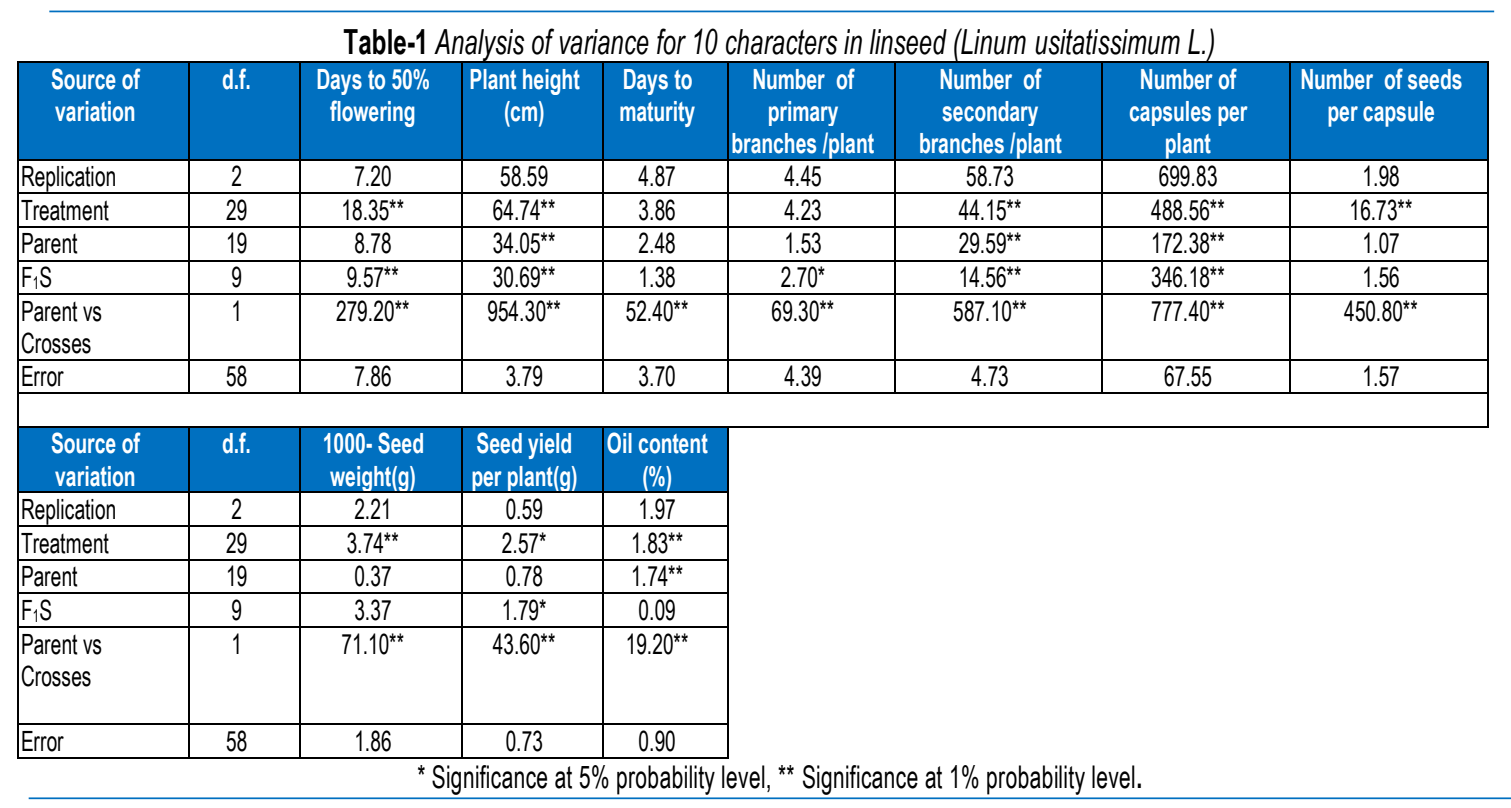

\section{Six parameter model}

The relative estimate of additive and non-additive gene action (dominance and epistasis interactions) based on six parameter model [Table-2] indicated the predominance of additive gene effects (d) in crosses RL-28-1 XL-53, NPHY 29 X LCK 87312 and NPHY 39 X NO 3 for days to 50\% flowering: NP 121 X RR 76, NPHY 29 X LCK 87312 and NPRR 402 X NP 8 for plant height (cm): RL-28-1 X L53, AHUDERA 170 XSJKO 05and NPHY 39 X NO 3 for days to maturity; NPHY 38 X LMHS-5and POLF 10 X SJKO 55 for number of primary branches per plant; NPHY 39 X NO 3 for number of secondary branches per plant; NPHY 38 X LMHS5, POLF 10 X SJKO 55 and NPHY 28 X 141N018XRR9 for capsules per plant; RL28-1 XL53, NPRR 271 X N0356 and NPHY 39 X NO 3 for number of seeds per capsules per plant;NPHY 29 X LCK 87312,and RL-28-1 X L-53 for 1000-seed weight (g); POLF 10 X SJKO 55, NPRR 402 X NP 8 and AHUDERA 170 X SJKO 05 for seed yield per plant and NPHY 29 X LCK 87312, NPRR 271 X NO 356 and NPHY 39 X NO 3 for oil content (\%). Such observation was reported by $[15,16]$. The predominance of dominance gene effect was observed in NPHY 29 X LCK 87312, POLF 10 X SJKO 55, AHUDERA 170 X SJKO 05, NPHY $28 X$ 141N018XRR9 and NP 121X RR 76 for late flowering, while five crosses was found with significant negative value for early flowering. Cross NPHY 38 X LMHS5 highly significant negative value for reduced plant height $(\mathrm{cm})$. However, crosses, NPHY 29 X LCK 87312, NP 121 X RR 76, NPRR 271 X No 356, NPRR 402 X NP 8, RL-2-1 X L-53 and AHUDERA 170 X SJKO 55 showed significant positive value for tallness. Crosses, NPRR 271 X No 356, NPRR 402 X NP 8, RL2-1 X L-53, NPHY 39 X N0 3, NPHY 28 X 141N018XRR9 and NPHY $38 X$ LMHS-5 highly significant negative value for early maturity, while crosses, NPHY
29 X LCK 87312, AHUDERA 170 X SJKO 05 and POLF 10 X SJKO 55 for late maturity. All crosses were highly positive significant for number of primary branches per plant. Crosses, NPHY 29 X LCK 87312, NP 121 X RR 76, NPRR 271 X No 356, NPRR 402 X NP 8, RL-28-1 X L-53 and AHUDERA 170 X SJKO 55, NPHY 39 X No 3 , NPHY 28 X 141N018XRR9 and NPHY 38 X LMHS-5 highly positive and significant for number of secondary branches per plant. All crosses except NPHY 39 X No 3 was highly positive and significant for number of capsules per plant. Cross NPHY 28 X 141N018XRR9 was found with significant negative value for number of seeds per capsule while crosses NPRR 271 X No 356 and RL-2-1 X L-53 were highly positive and significant for number of seeds per capsule. Crosses, 121 X RR 76, NPRR 402 X NP 8, AHUDERA 170 X SJKO 55, NPHY 38 X LMHS-5 and POLF $10 X$ SJKO was found with significant negative value for 1000-seed weight (g) while crosses NPRR 271 X No 356 and RL-28-1 X L-53 was highly positive and significant for 1000-seed weight(g). All crosses except NPHY 38 X LMHS-5 and POLF 10 X SJKO 55 was highly positive and significant seed yield per plant (g). All crosses except NP121 X RR76 was highly negative and significant for oil content (\%). Such observation was reported by [17, 18].

Among the components of epistatic interactions, additive $x$ additive (i) gene effect was more frequent followed by dominance $x$ dominance (I) and additive $x$ dominance (j) gene effect for most of the characters. The major role of additive $x$ additive gene effect was observed in variable number of crosses, viz., three crosses for days to $50 \%$ flowering, one cross for plant height $(\mathrm{cm})$, six crosses for early days to maturity, eight crosses for number of primary branches per plant, 
eight crosses for number of secondary branches per plant, nine crosses for number of capsules per plant, two crosses for number of seeds per capsule, two crosses for 1000-seed weight (g), seven crosses for seed yield per plant $(\mathrm{g})$ and one cross for oil content (\%). The role of additive $x$ dominance gene effect was observed in four crosses for early days to $50 \%$ flowering, five crosses for reduced plant height (cm), eight crosses for early days to maturity, two crosses for number of primary branches per plant, one cross for number of secondary branches per plant, two crosses for number of capsules per plant, two crosses for number of seeds per capsule, one cross for 1000-seed weight (g), nine crosses for seed yield per plant $(\mathrm{g})$ and five crosses for oil content (\%).

Table-2 Estimates of gene effect based on six parameters model for ten quantitative characters in linseed (Linum usitatissimum L.)

\begin{tabular}{|c|c|c|c|c|c|c|c|}
\hline \multirow[t]{2}{*}{ Crosses } & \multicolumn{6}{|c|}{ Genetic parameters } & \multirow{2}{*}{$\begin{array}{l}\text { Type of } \\
\text { epistasis }\end{array}$} \\
\hline & $m$ & $D$ & $H$ & I & $J$ & $L$ & \\
\hline \multicolumn{8}{|c|}{ Days to $50 \%$ flowering } \\
\hline NPHY 29 X LCK 87312 & $74.00^{* *}$ & $-3.66^{* *}$ & $44.66^{* *}$ & $44.66^{* *}$ & $-3.33^{* *}$ & $-61.33^{* *}$ & \multirow[t]{2}{*}{$D$} \\
\hline SE \pm & 0.24 & 0.48 & 1.50 & 1.38 & 0.62 & 2.47 & \\
\hline NP 121 XRR-76 & $75.00^{* *}$ & $4.33^{\star *}$ & $9.66^{* *}$ & $14.00^{* *}$ & $5.66^{* *}$ & $-8.66^{* *}$ & \multirow[t]{2}{*}{$D$} \\
\hline SE \pm & 0.24 & 0.39 & 1.35 & 1.25 & 0.54 & 2.10 & \\
\hline NPRR 271 X No 356 & $83.00^{* *}$ & 1.33 & $-21.83^{* *}$ & $-24.00^{* *}$ & -1.50 & $33.00^{* *}$ & \multirow[t]{2}{*}{ D } \\
\hline SE \pm & 0.24 & 0.65 & 1.89 & 1.63 & 0.94 & 3.38 & \\
\hline NPRR 402 X NP 8 & $81.00^{* *}$ & 0.33 & -0.66 & -0.66 & 0.33 & $-6.66^{* \star}$ & \multirow[t]{2}{*}{$C$} \\
\hline SE \pm & 0.24 & 0.48 & 1.43 & 1.38 & 0.57 & 2.31 & \\
\hline RL-28-1 XL-53 & $85.00^{* *}$ & $-4.33^{* *}$ & 0.66 & -0.66 & $-4.00^{\star *}$ & $-14.66^{* *}$ & \multirow[t]{2}{*}{$D$} \\
\hline SE \pm & 0.24 & 0.48 & 1.67 & 1.38 & 0.79 & 2.88 & \\
\hline AHUDERA X SJKO-05 & $76.00^{* *}$ & 0.00 & $21.83^{\star *}$ & $18.00^{* *}$ & $-10.16^{* *}$ & $-26.33^{* *}$ & \multirow[t]{2}{*}{$D$} \\
\hline SE \pm & 0.24 & 0.42 & 1.45 & 1.30 & 0.53 & 2.35 & \\
\hline NPHY 39 X No-3 & $83.00^{* *}$ & $-1.66^{* *}$ & $-25.16^{* *}$ & $-22.00^{* *}$ & 0.16 & $29.00^{* *}$ & \multirow[t]{2}{*}{$\mathrm{D}$} \\
\hline SE \pm & 0.24 & 0.54 & 1.74 & 1.46 & 0.87 & 3.05 & \\
\hline NPHY 28 X 141N018XRR9 & $76.00^{* *}$ & $-1.33^{*}$ & $14.83^{* *}$ & $14.66^{* *}$ & $-2.50^{\star *}$ & $-9.66^{* *}$ & \multirow[t]{2}{*}{$D$} \\
\hline SE \pm & 0.24 & 0.64 & 1.81 & 1.63 & 0.800 & 3.20 & \\
\hline NPHY-38 X LMHS-5 & $85.00^{* *}$ & $2.33^{* \star}$ & $-24.66^{* *}$ & $-27.33^{* \star}$ & 1.00 & $26.66^{* *}$ & \multirow[t]{2}{*}{$D$} \\
\hline SE \pm & 0.24 & 0.43 & 1.57 & 1.31 & 0.70 & 2.64 & \\
\hline POLF-6 X SJKO-5 & $73.00^{* *}$ & $5.00^{\star *}$ & $23.33^{* \star}$ & $23.33^{* *}$ & $5.33^{\star \star}$ & $-16.00^{\star \star}$ & \multirow[t]{2}{*}{ D } \\
\hline SE \pm & 0.24 & 0.44 & 1.62 & 1.32 & 0.88 & 2.76 & \\
\hline
\end{tabular}

Table-2 Contd.

\begin{tabular}{|c|c|c|c|c|c|c|c|}
\hline \multirow[t]{3}{*}{ Crosses } & \multicolumn{6}{|c|}{ Genetic parameters } & \multirow{2}{*}{$\begin{array}{l}\text { Type of } \\
\text { epistasis }\end{array}$} \\
\hline & $\bar{M}$ & d & $h$ & 1 & $J$ & L & \\
\hline & \multicolumn{6}{|c|}{ Plant height (cm). } & \\
\hline NPHY 29 X LCK 87312 & $45.86^{* *}$ & $-10.53^{* \star}$ & $29.00^{* *}$ & $38.40^{* *}$ & $-9.53^{* \star}$ & $-17.46^{* *}$ & \multirow[t]{2}{*}{$D$} \\
\hline SE \pm & 0.79 & 0.52 & 3.65 & 3.33 & 0.71 & 4.83 & \\
\hline NP 121 X RR-76 & $36.66^{* *}$ & $-13.33^{* \star}$ & $108.93^{\star \star}$ & $97.06^{\star \star}$ & $-24.06^{* *}$ & $-73.33^{* *}$ & \multirow[t]{2}{*}{$D$} \\
\hline SE \pm & 0.57 & 1.31 & 3.81 & 3.48 & 1.73 & 6.52 & \\
\hline NPRR 271 X No 356 & $49.13^{\star *}$ & 0.66 & $46.90^{* *}$ & $47.20^{\star *}$ & $6.90^{* *}$ & $-39.66^{* *}$ & \multirow[t]{2}{*}{ D } \\
\hline SE \pm & 0.71 & 1.41 & 4.44 & 4.02 & 2.19 & 7.37 & \\
\hline NPRR 402 X NP 8 & $43.80^{* *}$ & $-10.00^{* \star}$ & $77.40^{\star \star}$ & $62.66^{* *}$ & $-3.26^{\star *}$ & $-44.13^{* *}$ & \multirow[t]{2}{*}{$D$} \\
\hline SE \pm & 0.69 & 1.13 & 3.84 & 3.57 & 1.26 & 6.01 & \\
\hline RL-28-1 X L-53 & $50.40^{\star *}$ & $-2.06^{\star \star}$ & $32.43^{\star \star}$ & $8.93^{* \star}$ & $-4.10^{\star *}$ & $34.60^{* \star}$ & \multirow[t]{2}{*}{ C } \\
\hline SE \pm & 0.40 & 0.32 & 2.22 & 1.74 & 0.66 & 3.43 & \\
\hline AHUDERA X SJKO-05 & $76.00^{* *}$ & 0.00 & $21.83^{* *}$ & $18.00^{* *}$ & $-10.16^{* *}$ & $-26.33^{* *}$ & \multirow[t]{2}{*}{ D } \\
\hline SE \pm & 0.24 & 0.42 & 1.45 & 1.30 & 0.53 & 2.35 & \\
\hline NPHY 39 X No-3 & $62.06^{* \star}$ & $23.53^{\star \star}$ & 12.90 & 3.06 & $26.30^{* \star}$ & 7.26 & \multirow[t]{2}{*}{ C } \\
\hline SE \pm & 1.37 & 1.53 & 6.82 & 6.30 & 2.22 & 9.78 & \\
\hline NPHY 28 X 141N018XRR9 & $56.93^{\star *}$ & $6.33^{\star \star}$ & -2.63 & -7.60 & $12.96^{\star \star}$ & $20.46^{* \star}$ & \multirow[t]{2}{*}{$D$} \\
\hline SE \pm & 0.88 & 1.35 & 4.93 & 4.45 & 2.01 & 7.72 & \\
\hline NPHY-38 X LMHS-5 & $61.66^{* *}$ & $-3.46^{*}$ & $-14.26^{* *}$ & $-11.20^{\star *}$ & $5.93^{* *}$ & $33.60^{* *}$ & \multirow[t]{2}{*}{ D } \\
\hline SE \pm & 0.67 & 1.40 & 4.30 & 3.89 & 1.64 & 7.22 & \\
\hline POLF-6 X SJKO-5 & $66.53^{\star *}$ & $7.20^{* *}$ & -5.36 & -6.40 & $15.30^{* *}$ & -7.66 & \multirow[t]{2}{*}{ C } \\
\hline SE \pm & 0.72 & 1.85 & 4.80 & 4.70 & 1.89 & 8.20 & \\
\hline
\end{tabular}

\begin{tabular}{|c|c|c|c|c|c|c|c|}
\hline \multicolumn{8}{|c|}{ Table-2 Contd. } \\
\hline \multirow[t]{3}{*}{ Crosses } & \multicolumn{6}{|c|}{ Genetic parameters } & \multirow{2}{*}{$\begin{array}{l}\text { Type of } \\
\text { epistasis }\end{array}$} \\
\hline & $M$ & $d$ & $h$ & 1 & $j$ & $L$ & \\
\hline & \multicolumn{6}{|c|}{ Days to maturity } & \\
\hline NPHY 29 X LCK 87312 & $130.00^{* *}$ & -0.66 & $10.33^{* *}$ & $17.33^{\star *}$ & $-4.66^{* *}$ & $-56.66^{* *}$ & \multirow[t]{2}{*}{ D } \\
\hline SE \pm & 0.24 & 0.59 & 1.58 & 1.54 & 0.66 & 2.68 & \\
\hline NP 121 XRR-76 & $132.00^{* *}$ & $5.00^{* *}$ & 2.00 & $6.00^{* *}$ & $6.00^{* *}$ & $-32.00^{* *}$ & \multirow[t]{2}{*}{$\mathrm{D}$} \\
\hline SE \pm & 0.24 & 0.42 & 1.39 & 1.30 & 0.49 & 2.21 & \\
\hline NPRR 271 X No 356 & $137.00^{* *}$ & $2.00^{* *}$ & $-24.00^{* *}$ & $-24.00^{* *}$ & $-3.00^{* *}$ & $20.00^{* *}$ & \multirow[t]{2}{*}{$D$} \\
\hline SE \pm & 0.24 & 0.42 & 1.35 & 1.30 & 0.52 & 2.11 & \\
\hline NPRR 402 X NP 8 & $138.00^{* *}$ & $-6.00^{\star *}$ & $-15.50^{* *}$ & $-16.00^{* *}$ & $-3.50^{* \star}$ & $-5.00^{*}$ & \multirow[t]{2}{*}{ C } \\
\hline SE \pm & 0.24 & 0.42 & 1.35 & 1.30 & 0.52 & 2.11 & \\
\hline RL-28-1 XL-53 & $143.66^{* *}$ & $-13.00^{* *}$ & $-27.50^{* *}$ & $-28.66^{* *}$ & $-14.16^{* *}$ & $12.33^{* *}$ & \multirow[t]{2}{*}{ D } \\
\hline SE \pm & 0.31 & 0.42 & 1.56 & 1.50 & 0.55 & 2.27 & \\
\hline AHUDERA X SJKO-05 & $136.00^{* *}$ & $-11.00^{* *}$ & $15.50^{* *}$ & $14.00^{* *}$ & $-6.83^{* *}$ & $-59.00^{* *}$ & \multirow[t]{2}{*}{$\mathrm{D}$} \\
\hline SE \pm & 0.24 & 0.42 & 1.40 & 1.30 & 0.51 & 2.23 & \\
\hline NPHY 39 X No-3 & $135.00^{* *}$ & $-7.00^{* *}$ & $-7.16^{* *}$ & $-18.00^{* *}$ & $-9.83^{* *}$ & $14.33^{\star *}$ & $\mathrm{D}$ \\
\hline
\end{tabular}


Study on Generation Mean Analysis for Seed Yield and Its Associated Traits in linseed (Linum usitatissimum L.)

\begin{tabular}{|c|c|c|c|c|c|c|c|}
\hline SE \pm & 0.24 & 0.42 & 1.40 & 1.30 & 0.50 & 2.23 & \\
\hline NPHY 28 X 141N018XRR9 & $136.00^{* *}$ & $2.00^{* \star}$ & $-5.16^{* *}$ & $-8.00^{* \star}$ & $-1.83^{* \star}$ & $-13.66^{\star \star}$ & \multirow[t]{2}{*}{$C$} \\
\hline SE \pm & 0.24 & 0.54 & 1.52 & 1.47 & 0.65 & 2.53 & \\
\hline NPHY-38 X LMHS-5 & $143.00^{* *}$ & 0.66 & $-61.66^{* *}$ & $-54.66^{* *}$ & $-3.00^{* *}$ & $42.00^{* *}$ & \multirow[t]{2}{*}{$\mathrm{D}$} \\
\hline SE \pm & 0.24 & 0.38 & 1.31 & 1.24 & 0.47 & 2.01 & \\
\hline POLF-6 X SJKO-5 & $127.00^{* *}$ & $2.33^{* *}$ & $25.16^{* *}$ & $23.33^{* *}$ & $4.16^{* *}$ & $-50.33^{* *}$ & \multirow[t]{2}{*}{ D } \\
\hline SE \pm & 0.24 & 0.43 & 1.38 & 1.31 & 0.56 & 2.17 & \\
\hline
\end{tabular}

Table-2Contd.

\begin{tabular}{|c|c|c|c|c|c|c|c|}
\hline \multirow[t]{3}{*}{ Crosses } & \multicolumn{6}{|c|}{ Genetic parameters } & \multirow{2}{*}{$\begin{array}{l}\text { Type of } \\
\text { epistasis }\end{array}$} \\
\hline & M & $d$ & $h$ & 1 & j & $L$ & \\
\hline & \multicolumn{6}{|c|}{ Number of primary branches per plant } & \\
\hline NPHY 29 X LCK 87312 & $5.00^{\star \star}$ & $-2.00^{* *}$ & $7.16^{* *}$ & $8.00^{* *}$ & $-1.16^{* *}$ & $-18.33^{* \star}$ & \multirow[t]{2}{*}{$\mathrm{D}$} \\
\hline SE \pm & 0.24 & 0.33 & 1.26 & 1.19 & 0.48 & 1.86 & \\
\hline NP 121 X RR-76 & $3.66^{* \star}$ & 0.66 & $12.50^{\star \star}$ & $12.00^{* *}$ & -0.16 & $-22.33^{* *}$ & \multirow[t]{2}{*}{$\mathrm{D}$} \\
\hline SE \pm & 0.07 & 0.35 & 0.81 & 0.75 & 0.38 & 1.55 & \\
\hline NPRR 271 X No 356 & $3.33^{* *}$ & $-2.33^{* *}$ & $13.83^{* *}$ & $14.00^{* *}$ & $-2.50^{* *}$ & $27.66^{* *}$ & \multirow[t]{2}{*}{ C } \\
\hline SE \pm & 0.18 & 0.48 & 1.25 & 1.23 & 0.51 & 2.13 & \\
\hline NPRR 402 X NP 8 & $3.33^{* \star}$ & -0.33 & $8.16^{* *}$ & $10.00^{* *}$ & 0.50 & $-19.00^{* *}$ & \multirow[t]{2}{*}{ D } \\
\hline SE \pm & 0.18 & 0.35 & 1.07 & 1.02 & 0.46 & 1.71 & \\
\hline RL-28-1 X L-53 & $4.66^{* \star}$ & $-2.66^{\star *}$ & $11.00^{* *}$ & $10.66^{* *}$ & $-1.00^{* *}$ & $-16.66^{* *}$ & \multirow[t]{2}{*}{$D$} \\
\hline SE \pm & 0.35 & 0.17 & 1.50 & 1.46 & 0.30 & 1.71 & \\
\hline AHUDERA X SJKO-05 & $3.33^{* \star}$ & $-1.66^{*}$ & $21.16^{* *}$ & $18.00^{* *}$ & -1.16 & $-27.66^{* *}$ & \multirow[t]{2}{*}{$D$} \\
\hline SE \pm & 0.07 & 0.70 & 1.55 & 1.42 & 0.73 & 3.07 & \\
\hline NPHY 39 X No-3 & $6.66^{* \star}$ & 0.33 & $8.66^{* \star}$ & $-4.66^{* *}$ & -0.33 & $8.00^{* *}$ & \multirow[t]{2}{*}{ C } \\
\hline SE \pm & 0.35 & 0.39 & 1.63 & 1.62 & 0.43 & 2.63 & \\
\hline NPHY 28 X 141N018XRR9 & $5.00^{* *}$ & $-1.66^{* *}$ & $9.50^{* \star}$ & $7.33^{* *}$ & $-1.83^{* *}$ & $-13.66^{* *}$ & \multirow[t]{2}{*}{$\mathrm{D}$} \\
\hline SE \pm & 0.24 & 0.27 & 1.34 & 1.13 & 0.34 & 2.07 & \\
\hline NPHY-38 X LMHS-5 & $3.66^{* \star}$ & $6.33^{* *}$ & $28.50^{* *}$ & $23.33^{* *}$ & $5.83^{* \star}$ & $-35.66^{* \star}$ & \multirow[t]{2}{*}{$D$} \\
\hline SE \pm & 0.25 & 1.17 & 2.60 & 2.55 & 1.19 & 4.89 & \\
\hline POLF-6 X SJKO-5 & $6.66^{* \star}$ & $3.00^{* *}$ & $7.33^{\star \star}$ & $4.66^{*}$ & $4.33^{\star \star}$ & $-8.00^{*}$ & \multirow[t]{2}{*}{$\mathrm{D}$} \\
\hline SE \pm & 0.35 & 0.72 & 2.03 & 2.02 & 0.73 & 3.23 & \\
\hline
\end{tabular}

Table-2 Contd.

\begin{tabular}{|c|c|c|c|c|c|c|c|}
\hline \multirow[t]{3}{*}{ Crosses } & \multicolumn{6}{|c|}{ Genetic parameters } & \multirow{2}{*}{$\begin{array}{l}\text { Type of } \\
\text { epistasis }\end{array}$} \\
\hline & M & $d$ & $h$ & I & j & L & \\
\hline & \multicolumn{6}{|c|}{ Number of secondary branches per plant } & \\
\hline NPHY 29 X LCK 87312 & $9.00^{* *}$ & $-12.33^{* *}$ & $75.33^{* *}$ & $74.00^{* *}$ & $-10.00^{\star *}$ & $-96.66^{* *}$ & \multirow[t]{2}{*}{ D } \\
\hline SE \pm & 0.39 & 1.66 & 3.91 & 3.64 & 2.08 & 7.39 & \\
\hline NP 121 X RR-76 & $12.00^{* *}$ & $-10.00^{* *}$ & $41.00^{* *}$ & $33.33^{* *}$ & $-16.66^{* *}$ & $-22.00^{* *}$ & \multirow[t]{2}{*}{ D } \\
\hline SE \pm & 0.61 & 1.14 & 3.69 & 3.35 & 1.54 & -3.64 & \\
\hline NPRR 271 X No 356 & $9.66^{* *}$ & $-11.66^{* *}$ & $59.50^{* \star}$ & $60.66^{* \star}$ & $-6.83^{* *}$ & $-95.00^{* *}$ & \multirow[t]{2}{*}{ D } \\
\hline SE \pm & 0.25 & 0.74 & 2.49 & 1.81 & 1.63 & 4.66 & \\
\hline NPRR 402 X NP 8 & $9.00^{* *}$ & $-6.00^{\star *}$ & $62.16^{* *}$ & $69.33^{* *}$ & $-4.50^{\star *}$ & $-120.33^{* *}$ & \multirow[t]{2}{*}{ D } \\
\hline SE \pm & 0.44 & 1.39 & 3.47 & 3.30 & 1.70 & 6.22 & \\
\hline$R L-28-1 X L-53$ & $20.00^{* *}$ & $-9.33^{* *}$ & $41.00^{* *}$ & $33.33^{* *}$ & $-4.33^{\star \star}$ & $-23.33^{\star *}$ & \multirow[t]{2}{*}{ D } \\
\hline SE \pm & 1.21 & 0.46 & 5.13 & 4.93 & 0.66 & 5.90 & \\
\hline AHUDERA X SJKO-05 & $15.33^{* *}$ & $-6.00^{\star *}$ & $85.50^{* \star}$ & $76.00^{* *}$ & -0.16 & $-129.66^{* *}$ & \multirow[t]{2}{*}{ D } \\
\hline SE \pm & 0.82 & 1.94 & 5.23 & 5.07 & 2.24 & 8.78 & \\
\hline NPHY 39 X No-3 & $22.66^{* *}$ & $14.00^{* *}$ & $27.33^{* *}$ & 13.33 & $13.33^{* \star}$ & -24.00 & \multirow[t]{2}{*}{$\bar{D}$} \\
\hline SE \pm & 1.69 & 2.78 & 8.90 & 8.78 & 2.82 & 13.37 & \\
\hline NPHY 28 X 141N018XRR9 & $13.33^{* *}$ & $-6.33^{\star *}$ & $56.00^{* *}$ & $38.00^{* *}$ & $-6.66^{* *}$ & $-33.33^{* *}$ & \multirow[t]{2}{*}{$\mathrm{D}$} \\
\hline SE \pm & 0.82 & 0.80 & 3.81 & 3.65 & 1.03 & 5.08 & \\
\hline NPHY-38 X LMHS-5 & $22.00^{* *}$ & -1.00 & $72.50^{\star \star}$ & $43.33^{* \star}$ & 1.83 & $-67.66^{* *}$ & \multirow[t]{2}{*}{$D$} \\
\hline SE \pm & 1.27 & 2.59 & 7.51 & 7.28 & 2.81 & 12.14 & \\
\hline POLF-6 X SJKO-5 & $20.00^{* *}$ & 1.33 & 8.83 & 9.00 & 4.16 & -0.16 & \multirow[t]{2}{*}{$\bar{D}$} \\
\hline SE \pm & 0.97 & 2.18 & 5.92 & 9.72 & 2.31 & 15.41 & \\
\hline
\end{tabular}

Table-2 Contd.

\begin{tabular}{|c|c|c|c|c|c|c|c|}
\hline \multirow[t]{3}{*}{ Crosses } & \multicolumn{6}{|c|}{ Genetic parameters } & \multirow{2}{*}{$\begin{array}{l}\text { Type of } \\
\text { epistasis }\end{array}$} \\
\hline & M & $d$ & $h$ & 1 & j & L & \\
\hline & \multicolumn{6}{|c|}{ Number of capsules per plant } & \\
\hline NPHY 29 X LCK 87312 & $36.33^{* *}$ & $-42.00^{* *}$ & $254.00^{\star \star}$ & $249.33^{\star \star}$ & $-56.00^{* *}$ & $-297.33^{* *}$ & \multirow[t]{2}{*}{$\mathrm{D}$} \\
\hline SE \pm & 1.63 & 7.36 & 16.30 & 16.12 & 7.70 & 30.56 & \\
\hline NP 121 X RR-76 & $26.00^{* *}$ & $-64.00^{* *}$ & $267.33^{* \star}$ & $289.33^{* *}$ & $-69.33^{* *}$ & $-349.33^{* *}$ & \multirow[t]{2}{*}{ D } \\
\hline SE \pm & 1.24 & 5.26 & 14.32 & 11.65 & 5.90 & 27.30 & \\
\hline NPRR 271 X No 356 & $25.66^{* *}$ & $-62.00^{* *}$ & $333.00^{* \star}$ & $358.66^{* \star}$ & $-70.00^{* *}$ & $-543.33^{* *}$ & \multirow[t]{2}{*}{$\mathrm{D}$} \\
\hline $\mathrm{SE} \pm$ & 0.82 & 4.58 & 10.09 & 9.73 & 4.99 & 19.37 & \\
\hline NPRR 402 X NP 8 & $41.00^{* *}$ & $-18.33^{* *}$ & $234.83^{* \star}$ & $258.00^{* *}$ & $-22.50^{* *}$ & $-401.66^{* *}$ & \multirow[t]{2}{*}{ D } \\
\hline SE \pm & 2.52 & 2.69 & 12.15 & 11.44 & 3.98 & 16.88 & \\
\hline RL-28-1 X L-53 & $52.66^{* *}$ & $-76.66^{* *}$ & $383.33^{* \star}$ & $314.66^{* *}$ & $-58.66^{* *}$ & $-365.33^{* *}$ & \multirow[t]{2}{*}{$D$} \\
\hline SE \pm & 1.76 & 3.12 & 10.68 & 9.40 & 3.20 & 17.55 & \\
\hline AHUDERA X SJKO-05 & $37.33^{* *}$ & 3.33 & $559.66^{* \star}$ & $524.00^{* *}$ & -1.66 & $-814.00^{* *}$ & $\mathrm{D}$ \\
\hline
\end{tabular}


Yadav P. C., Yadav R. K., Dubey S.D., Singh H.C., Vishwanath, Pandey Yogesh and Kumar Sanjeev

\begin{tabular}{|c|c|c|c|c|c|c|c|}
\hline SE \pm & 1.99 & 5.14 & 15.31 & 13.01 & 5.76 & 27.32 & \multirow{3}{*}{ D } \\
\hline NPHY 39 X No-3 & $103.00^{* \star}$ & 19.66 & 51.83 & 18.00 & 17.83 & -2.33 & \\
\hline SE \pm & 6.96 & 10.92 & 36.48 & 35.39 & 11.10 & 54.74 & \\
\hline NPHY 28 X 141N018XRR9 & $59.66^{* \star}$ & $22.00^{*}$ & $286.33^{* *}$ & $250.66^{\star \star}$ & 22.66 & $-356.66^{\star \star}$ & \multirow[t]{2}{*}{ D } \\
\hline SE \pm & 3.61 & 9.84 & 25.89 & 24.43 & 10.22 & 45.30 & \\
\hline NPHY-38 X LMHS-5 & $53.33^{* \star}$ & $122.66^{* \star}$ & $456.33^{* *}$ & $353.33^{* \star}$ & $128.66^{* \hbar}$ & $-436.66^{\star \star}$ & \multirow[t]{2}{*}{ D } \\
\hline SE \pm & 3.56 & 8.76 & 24.39 & 22.57 & 9.35 & 42.10 & \\
\hline POLF-6 X SJKO-55 & $89.33^{* *}$ & $96.00^{* *}$ & $182.50^{* *}$ & $118.66^{* \star}$ & $102.50^{* *}$ & $-185.00^{* *}$ & \multirow[t]{2}{*}{$\mathrm{D}$} \\
\hline SE \pm & 3.77 & 3.34 & 18.61 & 16.51 & 3.75 & 26.49 & \\
\hline
\end{tabular}

\begin{tabular}{|c|c|c|c|c|c|c|c|}
\hline \multicolumn{8}{|c|}{ Table-2 Contd. } \\
\hline \multirow[t]{3}{*}{ Crosses } & \multicolumn{6}{|c|}{ Genetic parameters } & \multirow{2}{*}{$\begin{array}{l}\text { Type of } \\
\text { epistasis }\end{array}$} \\
\hline & $M$ & d & $h$ & 1 & j & L & \\
\hline & \multicolumn{6}{|c|}{ Number of seeds per capsule } & \multirow{3}{*}{ D } \\
\hline NPHY 29 X LCK 87312 & $6.66^{* *}$ & $0.66^{*}$ & -1.33 & 0.00 & 0.33 & 1.33 & \\
\hline SE \pm & 0.14 & 0.27 & 0.88 & 0.79 & 0.34 & 1.46 & \\
\hline NP 121 XRR-76 & $7.00^{* *}$ & $-0.66^{* *}$ & $-1.66^{*}$ & $-1.33^{*}$ & $-0.66^{*}$ & 0.66 & \multirow[t]{2}{*}{$\mathrm{D}$} \\
\hline SE \pm & 0.12 & 0.17 & 0.71 & 0.60 & 0.25 & 1.14 & \\
\hline NPRR 271 X No 356 & $6.33^{* *}$ & $0.66^{* *}$ & $4.00^{* *}$ & $4.00^{* *}$ & $0.66^{* \star}$ & -1.33 & \multirow[t]{2}{*}{$D$} \\
\hline SE \pm & 0.18 & 0.17 & 0.92 & 0.83 & 0.23 & 1.31 & \\
\hline NPRR 402 XNP 8 & $6.66^{* *}$ & $-1.33^{* *}$ & -0.83 & 0.00 & $-1.16^{* *}$ & $3.00^{*}$ & \multirow[t]{2}{*}{$\mathrm{D}$} \\
\hline SE \pm & 0.07 & 0.27 & 0.77 & 0.62 & 0.39 & 1.47 & \\
\hline RL-28-1 XL-53 & $5.66^{* *}$ & $1.33^{* *}$ & $2.83^{* *}$ & $2.66^{* *}$ & $1.50^{* *}$ & 1.00 & \multirow[t]{2}{*}{ C } \\
\hline SE \pm & 0.07 & 0.27 & 0.71 & 0.62 & 0.30 & 1.34 & \\
\hline AHUDERA 170 X SJKO-05 & $7.66^{* *}$ & $-0.66^{* \star}$ & $-2.16^{*}$ & $-2.66^{\star *}$ & -0.16 & $5.66^{* *}$ & \multirow[t]{2}{*}{$D$} \\
\hline SE \pm & 0.18 & 0.24 & 1.01 & 0.90 & 0.29 & 1.55 & \\
\hline NPHY 39 X No-3 & $7.00^{* *}$ & $0.66^{* *}$ & $-1.50^{*}$ & 1.66 & $1.50^{*}$ & -3.16 & \multirow[t]{2}{*}{$C$} \\
\hline SE \pm & 0.12 & 0.24 & 0.72 & 1.16 & 0.27 & 1.82 & \\
\hline NPHY 28 X 141N018XRR9 & $7.66^{* \star}$ & 0.33 & $-5.33^{* *}$ & $-6.00^{* *}$ & 0.33 & $14.66^{* *}$ & \multirow[t]{2}{*}{$\mathrm{D}$} \\
\hline SE \pm & 0.18 & 0.23 & 0.92 & 0.88 & 0.33 & 1.31 & \\
\hline NPHY-38 X LMHS-5 & $6.66^{* *}$ & $-1.00^{* *}$ & -1.16 & -0.66 & -0.50 & $6.33^{* *}$ & \multirow[t]{2}{*}{$\mathrm{D}$} \\
\hline SE \pm & 0.14 & 0.21 & 0.87 & 0.71 & 0.29 & 1.43 & \\
\hline POLF-6 X SJKO-55 & $7.33^{* \star}$ & -0.33 & $-4.33^{* *}$ & $-3.33^{* \star}$ & $-0.66^{*}$ & $7.33^{* k}$ & \multirow[t]{2}{*}{$D$} \\
\hline SEI & 0.14 & 0.29 & 1.01 & 0.81 & 0.34 & 1.77 & \\
\hline
\end{tabular}

\begin{tabular}{|c|c|c|c|c|c|c|c|}
\hline \multicolumn{8}{|c|}{ Table-2 Contd. } \\
\hline \multirow[t]{3}{*}{ Crosses } & \multicolumn{6}{|c|}{ Genetic parameters } & \multirow{2}{*}{$\begin{array}{l}\text { Type of } \\
\text { epistasis }\end{array}$} \\
\hline & M & d & $h$ & 1 & $j$ & L & \\
\hline & \multicolumn{6}{|c|}{ 1000-Seed weight (g) } & \multirow{3}{*}{ D } \\
\hline NPHY 29 X LCK 87312 & $6.79^{* *}$ & $0.43^{* *}$ & 0.28 & 0.22 & 0.07 & -0.43 & \\
\hline SE \pm & 0.04 & 0.16 & 0.41 & 0.38 & 0.21 & 0.74 & \\
\hline NP 121 XRR-76 & $6.85^{* *}$ & $-0.64^{* *}$ & $-2.11^{* *}$ & $-1.64^{* *}$ & -0.28 & $4.45^{* *}$ & \multirow[t]{2}{*}{$\bar{D}$} \\
\hline SEI & 0.03 & 0.20 & 0.44 & 0.43 & 0.21 & 0.85 & \\
\hline NPRR 271 X No 356 & $6.45^{* *}$ & $-0.60^{* *}$ & $1.03^{* *}$ & $0.76^{* *}$ & $-0.76^{* *}$ & 0.73 & \multirow[t]{2}{*}{ C } \\
\hline SEI & 0.04 & 0.10 & 0.33 & 0.27 & 0.15 & 0.57 & \\
\hline NPRR 402 XNP 8 & $6.90^{* *}$ & 0.20 & $-2.88^{* *}$ & $-2.06^{* *}$ & 0.26 & $3.93^{* *}$ & \multirow[t]{2}{*}{$D$} \\
\hline SE士 & 0.08 & 0.10 & 0.46 & 0.40 & 0.14 & 0.71 & \\
\hline$R L-28-1 X L-53$ & $6.63^{* *}$ & $0.33^{*}$ & $1.14^{* *}$ & $0.94^{* *}$ & $0.44^{* *}$ & $-1.72^{*}$ & \multirow[t]{2}{*}{$\bar{D}$} \\
\hline SEI & 0.04 & 0.14 & 0.44 & 0.34 & 0.19 & 0.81 & \\
\hline AHUDERA 170 X SJKO-05 & $6.77^{* *}$ & $-0.51^{* \star}$ & $-1.67^{* *}$ & $-1.28^{* *}$ & -0.25 & $2.05^{* *}$ & \multirow[t]{2}{*}{$\bar{D}$} \\
\hline SEI & 0.03 & 0.17 & 0.46 & 0.37 & 0.20 & 0.89 & \\
\hline NPHY $39 \times$ No-3 & $6.22^{* *}$ & -0.42 & -0.48 & -0.22 & $-0.60^{\star *}$ & $2.33^{* *}$ & \multirow[t]{2}{*}{$D$} \\
\hline SE \pm & 0.04 & 0.11 & 0.36 & 0.29 & 0.15 & 0.66 & \\
\hline NPHY 28 X 141N018XRR9 & $6.65^{* *}$ & $-0.55^{* *}$ & 0.12 & 0.26 & $-0.97^{\star *}$ & -0.27 & \multirow[t]{2}{*}{$D$} \\
\hline SEI & 0.05 & 0.18 & 0.52 & 0.43 & 0.21 & 0.97 & \\
\hline NPHY-38 X LMHS-5 & $6.92^{* *}$ & $-1.00^{* *}$ & $-3.58^{* *}$ & $-3.17^{* *}$ & $-1.69^{* \star}$ & $7.04^{* *}$ & \multirow[t]{2}{*}{$D$} \\
\hline SE \pm & 0.07 & 0.11 & 0.38 & 0.36 & 0.16 & 0.59 & \\
\hline POLF-6 X SJKO-55 & $12.30^{* \star}$ & $-0.47^{*}$ & $-23.67^{* *}$ & $-23.91^{\text {** }}$ & $-0.54^{*}$ & $26.28^{* *}$ & \multirow[t]{2}{*}{$\bar{D}$} \\
\hline SEI & 1.14 & 0.21 & 4.58 & 4.58 & 0.24 & 4.65 & \\
\hline
\end{tabular}

\begin{tabular}{|c|c|c|c|c|c|c|c|}
\hline \multirow[t]{3}{*}{ Crosses } & \multicolumn{6}{|c|}{ Genetic parameters } & \multirow{2}{*}{ Type of epistasis } \\
\hline & M & d & $h$ & 1 & j & L & \\
\hline & \multicolumn{6}{|c|}{ Seed yield per plant (g) } & \multirow{3}{*}{ D } \\
\hline NPHY 29 X LCK 87312 & $4.39^{* *}$ & $1.46^{* *}$ & $8.01^{* *}$ & $6.80^{* *}$ & $0.96^{* *}$ & $-6.40^{* *}$ & \\
\hline SEI & 0.10 & 0.17 & 0.58 & 0.54 & 0.26 & 0.91 & \\
\hline NP 121 XRR-76 & $3.82^{* *}$ & $0.30^{* *}$ & $11.48^{* *}$ & $9.28^{* *}$ & 0.08 & $-11.96^{* \star}$ & \multirow[t]{2}{*}{$D$} \\
\hline SE \pm & 0.21 & 0.09 & 0.89 & 0.87 & 0.18 & 1.03 & \\
\hline NPRR 271 X No 356 & $5.21^{* \star}$ & $1.75^{* *}$ & $4.79^{* *}$ & $2.92^{* *}$ & $2.05^{\star *}$ & $-2.89^{* *}$ & \multirow[t]{2}{*}{$\bar{D}$} \\
\hline SE \pm & 0.11 & 0.03 & 0.50 & 0.45 & 0.20 & 0.62 & \\
\hline NPRR 402 XNP 8 & $4.75^{\star \star}$ & $2.42^{* *}$ & $4.77^{* *}$ & $4.14^{* *}$ & $2.86^{* *}$ & $-2.36^{* *}$ & \multirow[t]{2}{*}{$D$} \\
\hline SEI & 0.16 & 0.12 & 0.75 & 0.70 & 0.23 & 0.96 & \\
\hline$R L-28-1 X L-53$ & $4.37^{* \star}$ & $1.22^{* *}$ & $8.14^{* *}$ & $7.09^{* \star}$ & $2.38^{* *}$ & $-7.43^{* *}$ & $D$ \\
\hline
\end{tabular}




\begin{tabular}{|c|c|c|c|c|c|c|c|}
\hline SE \pm & 0.15 & 0.25 & 0.81 & 0.80 & 0.27 & 1.22 & \\
\hline AHUDERA 170 X SJKO-05 & $3.40^{\star \star}$ & $2.08^{* *}$ & $12.75^{\star *}$ & $10.37^{\star \star}$ & $2.89^{* *}$ & -9.90 & \multirow[t]{2}{*}{$D$} \\
\hline SE \pm & 0.06 & 0.08 & 0.35 & 0.31 & 0.14 & -18.79 & \\
\hline NPHY $39 \times$ No-3 & $4.77^{\star \star}$ & $2.04^{* *}$ & $5.38^{\star \star}$ & $4.28^{\star *}$ & $2.92^{* *}$ & -1.56 & \multirow[t]{2}{*}{$D$} \\
\hline SEI & 0.04 & 0.17 & 0.43 & 0.38 & 0.18 & 0.82 & \\
\hline NPHY $28 X 141$ N018XRR9 & $5.57^{\star \star}$ & $1.19^{\star \star}$ & $2.62^{* *}$ & $1.13^{*}$ & $1.21^{* *}$ & $3.09^{* *}$ & \multirow[t]{2}{*}{ C } \\
\hline SE \pm & 0.11 & 0.15 & 0.57 & 0.53 & 0.24 & 0.85 & \\
\hline NPHY-38 X LMHS-5 & $5.89^{\star \star}$ & $1.21^{* \star}$ & 0.72 & -0.46 & $1.63^{* *}$ & $2.63^{* *}$ & \multirow[t]{2}{*}{$\mathrm{C}$} \\
\hline SE \pm & 0.09 & 0.08 & 0.45 & 0.39 & 0.23 & 0.65 & \\
\hline POLF-6 X SJKO-55 & $5.53^{\star \star}$ & $2.70^{* *}$ & $1.05^{\star}$ & 0.53 & $2.78^{* *}$ & $4.60^{* *}$ & \multirow[t]{2}{*}{$C$} \\
\hline SE \pm & 0.104 & 0.106 & 0.47 & 0.46 & 0.12 & 0.63 & \\
\hline
\end{tabular}

Table-2 Contd.

\begin{tabular}{|c|c|c|c|c|c|c|c|}
\hline \multirow[t]{3}{*}{ Crosses } & \multicolumn{6}{|c|}{ Genetic parameters } & \multirow{2}{*}{$\begin{array}{r}\text { Type of } \\
\text { epistasis }\end{array}$} \\
\hline & $M$ & $d$ & $h$ & I & $j$ & $L$ & \\
\hline & \multicolumn{6}{|c|}{ Oil content (\%) } & \multirow{3}{*}{$D$} \\
\hline NPHY 29 X LCK 87312 & $35.83^{* *}$ & $2.47^{\star *}$ & $-3.14^{* *}$ & $-2.88^{* *}$ & $3.01^{* \star}$ & $8.39^{* \star}$ & \\
\hline SE \pm & 0.11 & 0.08 & 0.50 & 0.47 & 0.17 & 0.66 & \\
\hline NP 121 XRR-76 & $34.48^{* *}$ & $0.53^{\star \star}$ & 0.26 & $5.23^{\star *}$ & 0.24 & $-12.03^{* *}$ & \multirow[t]{2}{*}{$D$} \\
\hline SE \pm & 0.15 & 0.07 & 0.74 & 0.63 & 0.39 & 1.04 & \\
\hline NPRR 271 X No 356 & $36.38^{* *}$ & $2.74^{* \star}$ & $-5.55^{\star \star}$ & $-3.96^{\star \star}$ & $3.70^{* *}$ & $13.03^{* *}$ & \multirow[t]{2}{*}{$\bar{D}$} \\
\hline SE \pm & 0.08 & 0.07 & 0.42 & 0.35 & 0.25 & 0.65 & \\
\hline NPRR 402 XNP 8 & $35.57^{\text {** }}$ & $2.04^{* *}$ & -0.62 & $-1.72^{\star *}$ & $3.36^{* *}$ & $3.65^{\star \star}$ & \multirow[t]{2}{*}{$D$} \\
\hline SE \pm & 0.15 & 0.07 & 0.67 & 0.62 & 0.25 & 0.83 & \\
\hline$R L-28-1 X L-53$ & $36.22^{* *}$ & $-2.51^{* *}$ & $-5.91^{* *}$ & -0.33 & $-1.11^{\star *}$ & -0.01 & \multirow[t]{2}{*}{ C } \\
\hline SE \pm & 0.04 & 0.07 & 0.57 & 0.21 & 0.23 & 1.12 & \\
\hline AHUDERA 170 X SJKO-05 & $36.48^{* *}$ & $-1.27^{\star \star}$ & $-22.82^{* *}$ & $-21.15^{\star \star}$ & $-1.40^{\star \star}$ & $40.35^{\star \star}$ & \multirow[t]{2}{*}{$D$} \\
\hline SE士 & 0.09 & 0.07 & 0.44 & 0.38 & 0.21 & 0.62 & \\
\hline NPHY 39 X No-3 & $36.65^{* \star}$ & $1.93^{\star \star}$ & $-13.16^{* *}$ & $-11.31^{* *}$ & $3.38^{* \star}$ & $21.84^{\star *}$ & \multirow[t]{2}{*}{$\bar{D}$} \\
\hline SE \pm & 0.05 & 0.05 & 0.48 & 0.25 & 0.30 & 0.88 & \\
\hline NPHY $28 \times 141 N 018 X R R 9$ & $35.88^{* *}$ & $1.01^{* *}$ & $-11.70^{* *}$ & $-8.79^{\star \star}$ & 0.47 & $15.83^{* *}$ & \multirow[t]{2}{*}{$\bar{D}$} \\
\hline SE \pm & 0.10 & 0.11 & 0.56 & 0.48 & 0.23 & 0.84 & \\
\hline NPHY-38 X LMHS-5 & $35.58^{* *}$ & $0.68^{* *}$ & $-5.78^{\star \star}$ & $-5.08^{\star \star}$ & $2.32^{\star \star}$ & $10.26^{* *}$ & \multirow[t]{2}{*}{$\bar{D}$} \\
\hline SE \pm & 0.05 & 0.07 & 0.34 & 0.26 & 0.21 & 0.57 & \\
\hline POLF-6 X SJKO-55 & $36.73^{\star *}$ & $0.66^{* *}$ & $-9.55^{\star *}$ & $-6.48^{* \star}$ & $0.48^{*}$ & $8.11^{* *}$ & \multirow[t]{2}{*}{ D } \\
\hline SE \pm & 0.04 & 0.12 & 0.34 & 0.29 & 0.20 & 0.62 & \\
\hline
\end{tabular}

The role of dominance $x$ dominance gene effect was observed in seven crosses for early days to $50 \%$ flowering, five crosses for reduced plant height (cm), five crosses for early days to maturity, two crosses for number of primary branches per plant, none of the cross for number of secondary branches per plant, none of the cross for number of capsules per plant, four crosses for number of seeds per capsule, six crosses for 1000 -seed weight (g), three crosses for seed yield per plant and eight crosses for oil content (\%).

\section{Heterosis and inbreeding depression}

The computed value of heterosis over better and mid parent [Table-3] revealed that none of the crosses showed useful significant heterosis for all the characters. The degree and direction of heterosis varied not only from character to character but also from cross to cross. In general considerable amount of significant desirable mid parent heterosis was reported in two crosses for days to $50 \%$ flowering, one cross for plant height (cm), two crosses for days to maturity, two crosses for number of primary branches per plant, five crosses for number of secondary branches per plant, four crosses for number of capsules per plant, none of the cross combination showed positive significant heterosis for number of seeds per capsule, none of the cross combination showed positive significant heterosis for 1000-seed weight (g), six crosses for seed yield per plant $(\mathrm{g})$ and one cross for oil content (\%). As regards to heterosis over better parent, significant and desirable heterosis was observed for all the character except number of seeds per capsule and 1000-seed weight (g).Significant and desirable heterosis was observed in one cross for days to $50 \%$ flowering, none of the cross combination showed positive significant heterosis for plant height $(\mathrm{cm})$, three crosses for days to maturity, two crosses for number primary branches per plant, five crosses for number of secondary branches per plant, three crosses for number of capsules per plant, none of the cross combination showed positive significant heterosis for number of seeds per capsule and 1000-seed weight (g), two crosses for seed yield per plant $(\mathrm{g})$, none of the cross combination showed positive significant heterosis for oil content $(\%)$.
While considering the significance and useful heterosis over better parent and mid parent [Table-2] the best crosses. viz., NPHY 29 X LCK 87312, NP 121 X RR 76, NPRR 271 X No 3, RL-28-1 X L-53, AHUDERA 170 X SJKO 55 and NPHY 39 X No 3 for seed yield per plant $(\mathrm{g})$ were considered. It was found that increase in seed yield in almost all these crosses is due to desirable heterosis of important components traits like number of primary branches per plant, number of secondary branches per plant, number of capsules per plant, number of seeds per capsule and 1000-seed weight $(\mathrm{g})$. The contribution of these components to the heterosis for seed yield per plant. Heterotic response in respected of seed yield per plant was observed by workers $[19,20]$.

In the present cropping scenario there is an urgent need to take up linseed breeding programme to develop early maturing verities in this Annual Report AICLP (2014) NPHY 29 X LCK 87312, NP 121 X RR 76, NP271 X NO 356 and AHUDERA 170 X SJKO 05 significant hybrids for days to $50 \%$ flowering Out of 10 crosses only one cross (NP 121 X RR 76) showed highly significant negative heterosis for early flowering and Such observations were reported by [21-23]. Oil content is an important character and the basic aim of linseed breeding is to increase oil producing either by increase oil content or seed yield or both out of 10 crosses only one cross NPRR 402 X NP 8 showed significant positive better heterosis for oil content (\%) and Out of 10 crosses none of them showed significant positive mid parent heterosis for oil content(\%).

Eight crosses Namely, NPHY 29 X LCK 87312, NP121 X RR 76, NPRR271 X NO 356, RL-28-1 X L-53, AHUDER 170 X SJKO 05, NPHY 39 X NO 3, NPHYNPHY 28 X 141N018XRR9 and POLF $10 X$ SJKO 55 show non-significant positive better parent heterosis for seed yield per plant and Out of 10 crosses, NHY $29 X$ LCK 87312, NP121 X RR 76, NPRR271 X NO 356, RL-28-1 X L-53, AHUDERA 170 X SJKO 05, NPHY 39 X NO 3, NPHY 28 X 141N018XRR9 and NPHYB $38 X$ LMHS-5 showed significant positive mid parent heterosis for seed yield per plant (g). Therefore, there is need to screen out genotypes possessing more oil content in the crop for further exploitation of heterosis. Limited heterotic response for oil content has been reported by [24] observed that the combinations giving 
pronounced heterosis in $\mathrm{F}_{1}$ should high inbreeding depression in $\mathrm{F}_{2}$ indicating non- $\quad$ allelic gene interactions in high self pollinated crop obtained.

Table-3 Estimates of heterosis over better parents, mid parent and inbreeding depression in percent for 10 characters in linseed (Lin um usitatissimum L.)

\begin{tabular}{|c|c|c|c|c|c|c|c|c|c|}
\hline \multirow[t]{2}{*}{ Crosses combinations } & \multicolumn{3}{|c|}{ Days to $50 \%$ flowering } & \multicolumn{3}{|c|}{ Plant height (cm) } & \multicolumn{3}{|c|}{ Days to maturity } \\
\hline & $B P$ & MP & ID & $B P$ & MP & ID & $B P$ & MP & ID \\
\hline NPHY 29 X LCK 87312 & -0.41 & 0.00 & 8.64 & $-15.66^{*}$ & -14.37 & 18.09 & $-8.33^{* \star}$ & $-5.46^{\star \star}$ & -7.43 \\
\hline NP 121 XRR-76 & $-6.80^{* \star}$ & $-5.28^{*}$ & 3.43 & $45.02^{* *}$ & $19.47^{* \star}$ & 49.63 & $-3.84^{*}$ & $-3.10^{*}$ & -5.60 \\
\hline NPRR 271 X No 356 & -0.82 & 2.77 & -3.31 & 10.45 & -0.47 & 21.59 & $-3.70^{*}$ & 0.00 & -5.38 \\
\hline NPRR 402XNP 8 & 0.00 & 0.00 & -2.53 & $42.93^{* *}$ & $25.96^{* \star}$ & 38.71 & -1.52 & 0.38 & -6.97 \\
\hline$R L-28-1 X L-53$ & 1.24 & 1.66 & -4.08 & $39.90^{* *}$ & $45.39^{* \star}$ & 33.03 & 1.78 & 0.88 & -8.02 \\
\hline AHUDERA 170 X SJKO-05 & $6.63^{* \star}$ & $5.01^{*}$ & 5.39 & -7.02 & 12.10 & 26.45 & $-2.02^{*}$ & 1.17 & -5.42 \\
\hline NPHY 39 X No-3 & -6.04 & -3.91 & -6.86 & 11.17 & 16.25 & 11.75 & $6.29^{\star *}$ & $8.72^{\star \star}$ & 0.00 \\
\hline NPHY 28 X 141N018XRR9 & -1.22 & 0.20 & 6.17 & 23.60 & 8.90 & 6.25 & -0.76 & 2.22 & -4.61 \\
\hline NPHY-38 X LMHS-5 & 1.70 & 3.47 & -7.14 & 11.19 & -4.64 & 2.01 & $-2.64^{*}$ & $-5.39^{\star \star}$ & -16.57 \\
\hline POLF-6 X SJKO-5 & -0.41 & 0.00 & 9.50 & 17.29 & 1.69 & -7.42 & $2.93^{*}$ & 1.46 & 0.00 \\
\hline
\end{tabular}

\begin{tabular}{|c|c|c|c|c|c|c|c|c|c|}
\hline \multirow[t]{2}{*}{ Crosses combination } & \multicolumn{3}{|c|}{$\begin{array}{l}\text { Number of primary branches } \\
\text { per plant }\end{array}$} & \multicolumn{3}{|c|}{ Number of secondary branches } & \multicolumn{3}{|c|}{ Number of capsules per plant } \\
\hline & $B P$ & $M P$ & ID & $B P$ & MP & ID & $B P$ & MP & ID \\
\hline NPHY 29 X LCK 87312 & 0.00 & -17.24 & -25.00 & 18.96 & 6.15 & 60.87 & 26.54 & 5.53 & 59.17 \\
\hline NP121 XRR-76 & 44.44 & 13.04 & 15.38 & $113.15^{* *}$ & 39.65 & 55.55 & -18.72 & -23.32 & 64.05 \\
\hline NPRR 271 X No 356 & -9.09 & -4.76 & 0.00 & 30.55 & -6.93 & 38.29 & $-37.40^{*}$ & $-31.30^{*}$ & 54.43 \\
\hline NPRR 402 XNP 8 & -27.27 & -40.74 & -25.00 & $-46.42^{\star}$ & $-41.74^{*}$ & 10.00 & -24.67 & -28.54 & 29.31 \\
\hline$R L-28-1 \times L-53$ & 50.00 & 5.88 & 22.22 & $57.57^{\star *}$ & $28.39^{*}$ & 42.30 & $49.51^{\star \star}$ & $81.42^{* *}$ & 65.57 \\
\hline $\begin{array}{l}\text { AHUDERA } 170 \text { X SJKO- } \\
05\end{array}$ & $110.00^{* *}$ & 82.60 & 52.38 & $148.38^{\text {t* }}$ & $58.76^{\text {** }}$ & 40.26 & $55.70^{* *}$ & $45.72^{\star}$ & 67.15 \\
\hline NPHY $39 \times$ XNo-3 & $85.71^{\star}$ & $116.66^{*}$ & 23.07 & $93.61^{* *}$ & $85.71^{* *}$ & 25.27 & $38.48^{*}$ & $35.80^{*}$ & 19.74 \\
\hline $\begin{array}{l}\text { NPHY } 28 X \\
\text { 141N018XRR9 }\end{array}$ & 58.33 & 52.00 & 21.05 & $125.00^{* *}$ & $120.00^{* *}$ & 59.59 & $46.35^{*}$ & $45.72^{*}$ & 47.50 \\
\hline NPHY-38 X LMHS-5 & $170.00^{* *}$ & $134.78^{* *}$ & 59.25 & $342.85^{\text {t* }}$ & $239.72^{* *}$ & 46.77 & $172.10^{* *}$ & $148.55^{* *}$ & 69.05 \\
\hline POLF- 6 X SJKO-5 & $92.30^{* \star}$ & 47.05 & 20.00 & $77.77^{* *}$ & $49.53^{* *}$ & 25.00 & $109.89^{* *}$ & $90.54^{* *}$ & 33.49 \\
\hline
\end{tabular}

\begin{tabular}{|c|c|c|c|c|c|c|c|c|c|}
\hline \multicolumn{10}{|c|}{ Table-3 Contd. } \\
\hline \multirow[t]{2}{*}{ Crosses combinations } & \multicolumn{3}{|c|}{ Number of seeds per capsule } & \multicolumn{3}{|c|}{ 1000- Seed weight (g) } & \multicolumn{3}{|c|}{ Seed yield per plant (g) } \\
\hline & $B P$ & MP & ID & $B P$ & MP & ID & $B P$ & MP & ID \\
\hline NPHY 29 X LCK 87312 & -13.63 & -17.39 & -5.26 & 6.49 & 0.83 & 0.48 & $33.59^{* \star}$ & $21.79^{\star}$ & 35.39 \\
\hline NP 121XRR-76 & -5.00 & -5.00 & -10.52 & -1.51 & -6.32 & 0.82 & $43.41^{* *}$ & $50.42^{* \star}$ & 41.83 \\
\hline NPRR 271 X No 356 & 0.00 & 0.00 & 20.83 & 6.49 & 3.94 & 9.78 & $46.14^{* \star}$ & $37.16^{\star}$ & 24.31 \\
\hline NPRR 402 XNP 8 & -8.69 & -10.63 & 4.76 & -10.55 & -11.22 & -7.08 & 19.38 & 10.51 & 27.37 \\
\hline$R L-28-1 X L-53$ & 4.76 & 2.32 & 22.72 & 5.88 & 3.17 & 2.11 & $50.57^{\star \star}$ & $18.95^{*}$ & 33.58 \\
\hline $\begin{array}{l}\text { AHUDERA } 170 \text { X SJKO- } \\
05\end{array}$ & 14.28 & 6.66 & 4.16 & -2.02 & -5.66 & -5.01 & $27.36^{* \star}$ & $48.30^{* *}$ & 53.37 \\
\hline NPHY $39 \times$ No-3 & $-25.92^{* *}$ & $-18.36^{\star}$ & -5.00 & -1.19 & -3.84 & 5.08 & $38.80^{* *}$ & $18.29^{*}$ & 32.53 \\
\hline NPHY $28 X 141$ N018XRR9 & 8.33 & 8.33 & 11.53 & 4.40 & -2.08 & -0.10 & $24.43^{*}$ & $24.09^{*}$ & 27.20 \\
\hline NPHY-38 X LMHS-5 & -11.53 & -6.12 & 13.04 & 4.08 & -5.66 & -0.48 & $30.40^{*}$ & $20.73^{\star}$ & 14.78 \\
\hline POLF-6 X SJKO-5 & -8.69 & -12.50 & -4.76 & 2.37 & 3.53 & -74.84 & 9.07 & 7.77 & 23.25 \\
\hline
\end{tabular}

\begin{tabular}{|c|c|c|c|}
\hline \multirow{2}{*}{ Crosses combinations } & \multicolumn{3}{|c|}{ Oil content (\%) } \\
\hline & $B P$ & MP & ID \\
\hline NPHY 29 X LCK 87312 & 0.75 & -0.72 & 1.44 \\
\hline NP121 XRR-76 & $-12.91^{\text {t* }}$ & $-13.59^{* *+}$ & -9.16 \\
\hline NPRR 271 X No 356 & $-6.48^{\star \star}$ & $-4.13^{*}$ & 1.31 \\
\hline NPRR 402 XNP 8 & $7.16^{* *}$ & 3.13 & 1.65 \\
\hline$R L-28-1 X L-53$ & $-11.18^{* \star}$ & $-14.37^{* \star *}$ & -8.90 \\
\hline AHUDERA $170 \mathrm{X}$ SJKO-05 & $-4.19^{*}$ & $-4.54^{*}$ & -3.77 \\
\hline NPHY 39 X No-3 & $-8.49^{* *}$ & $-4.94^{*}$ & -3.15 \\
\hline NPHY 28 X 141N018XRR9 & $-6.52^{* \star}$ & $-7.89^{* *}$ & -5.56 \\
\hline NPHY-38X LMHS-5 & 2.72 & -1.95 & -0.92 \\
\hline POLF-6X XJKO-5 & $-7.87^{\star \star}$ & $-8.30^{* *}$ & -8.05 \\
\hline
\end{tabular}

\section{Heritability and genetic advance}

High heritability was observed in [Table-4] characters like number of capsules per plant, 1000-seed weight $(\mathrm{g})$ and seed yield per plant $(\mathrm{g})$. whereas medium heritability was observed in characters like plant height $(\mathrm{cm})$, days to maturity, number of primary branches per plant, number of secondary branches per plant and number of seeds per capsule, whereas low heritability was observed in characters like days to $50 \%$ flowering and oil content (\%)In $\mathrm{F}_{1}$ generation characters likes number of seeds per capsule. exhibited high value of genetic advance, whereas, number of secondary branches per plant, and oil content (\%). exhibited moderate value of genetic advance, whereas, days to $50 \%$ flowering, plant height $(\mathrm{cm})$, number of primary branches per plant, number of capsules per plant, 1000-seed weight $(\mathrm{g})$ and seed yield per plant $(\mathrm{g})$ exhibited low genetic advance. Such observations were reported by $[25,26]$.

\section{Conclusion}

The results of heritability coupled with genetic advance in percent of mean 
showed that none of the characters had moderate heritability along with high genetic advance; Rest of the traits had low to high heritability with low to moderate

genetic gain in generations.

Table-4 Estimate of heritability, genetic advance and genetic advance in percent of mean for 10 characters in linseed (Linum usitatissimum L.)

\begin{tabular}{|l|c|c|c|c|c|}
\hline \multicolumn{1}{|c|}{ Characters } & Heritability & \multicolumn{2}{c|}{ Population mean } & Genetic advance & $\begin{array}{c}\text { Genetic advance in } \\
\text { percent of mean }\end{array}$ \\
\cline { 3 - 5 } & & $F 1$ & $F 2$ & -7.46 & -9.38 \\
\hline Days to 50\% flowering & -0.83 & 79.86 & 79.10 & -4.06 & -6.91 \\
\hline Plant hight (cm) & -0.01 & 65.58 & 51.84 & -0.76 & -0.57 \\
\hline Days to maturity & -0.10 & 128.16 & 135.76 & 0.14 & 3.67 \\
\hline No of primary branches /plant & 10.17 & 5.96 & 4.52 & 3.61 & 18.01 \\
\hline $\begin{array}{l}\text { No of secondary branches } \\
\text { /plant }\end{array}$ & 2.37 & 24.79 & 15.29 & & 6.52 \\
\hline No of capsules per plant & 4.99 & 109.09 & 57.96 & 5.45 & 30.21 \\
\hline No of seeds per capsule & 1.22 & 7.29 & 6.82 & 2.13 & 2.85 \\
\hline 1000- Seed weight & 5.62 & 6.77 & 7.25 & 0.20 & 1.89 \\
\hline Seed yield per plant & 0.47 & 6.95 & 4.77 & 0.11 & 16.19 \\
\hline Oil content (\%) & 7.40 & 34.81 & 35.98 & 5.73 & \\
\hline
\end{tabular}

Application of research: Study on Generation Mean Analysis for Seed Yield

Research Category: Genetics and Plant Breeding

\section{Abbreviations:}

ANOVA: Analysis of variance

Acknowledgement / Funding: Author are thankful to Chandra Shekhar Azad University of Agriculture and Technology, Kanpur 208002, Uttar Pradesh

\section{*Major advisor: Dr R K Yadav}

University: Chandra Shekhar Azad University of Agriculture and Technology, Kanpur, 208002

Research project name or number: Ph. D Thesis

Author Contributions: All author equally contributed

Author statement: All authors read, reviewed, agree and approved the final manuscript

\section{Conflict of Interest: None declared}

Ethical approval: This article does not contain any studies with human participants or animals performed by any of the authors

\section{References}

[1] Anonymous (2014-2015) Food and Agriculture organization of the United Nation. FAO Statistics. (www.faostat.org.com).

[2] Doucet I. and Filispescu H. (1981) Analelelnstituty de certaruOebtrycereake Si PlanteTehniceFundul, 46, 35-48.

[3] Singh A. and Tewari N. (2014) J. Oilseeds Res., 31(2), 118-122.

[4] Hayman B.I. (1958a) Genetics, 43, 63-64.

[5] Jinks J.L. and Jones J.M. (1958) Genetic, 43, 223-234.

[6] Anonymous (1975) methods of analysis, $12^{\text {th }}$ edition, Association of official analytical chemists, Washington, D.C.

[7] Luddy E.F., Barforad R.A., Berbs S.F. and Magidman P. (1968) J Amer Oil ChemSoc, 65, 549.

[8] Kashyap O.P. and Rastogi N.K. (2006) Annals of Agricultural Research, 27(2), 103-110.

[9] Wang Yu Fu, Yan Zhongfeng, Fan Jian Qiaoguangjun, Lu yun, Wu Guangwen, Wang Diankui and Yang Lijun Wang Yan hua (1996) China's Fibre Crops, 2, 10-13.

[10] Yadav R.K. and Srivastava S.B.L. (2002) Crop Res., 23(2), 277-282.

[11] Anand J.J. Rana B.S. and Jain O.P. (1972) News Letter 4, (1), 33-37.

[12] Verma A.K. and Sinha P.K. (1993) Heterosis in linseed. In heterosis breeding in crop plants. Theory and application: short communication: symposium Ludhiana, 23-24 Feb. 1993.
[13] Rowr P.R. and Andrew R.H. (1964) Crop Sci., 4, 563-567.

[14] Vishnu A., Shah M.A. and Lakshya Deep (2005) Electronic journal of Plant Breeding, 5(1),127-129.

[15] Singh P., Singh D. and Singh S.K. (2005) Farm Science Journal, 14(2), 1-3.

[16] Chauhan V.S., Kumar M.P. and Singh R.B. (2006) Farm Science Journal, 15(1), 29-31.

[17] Tripathi S., Mishra V. and Tripathi H.C. (2011) New Agriculturist, 22(2), 207-210.

[18] Rao D.G. and Pandagare S.S.(2011) Asian Journal of Bio Science, 6(1), 16-22.

[19] Kumar S., Kumar R., Kumar S., Singh S., Kumar S. and Singh M.P. (2014) International Journal of Agricultural Sciences, 10(1), 356-359.

[20] Yadav R. K. (2000) Plant Archives, 1(1\&2), 95-98.

[21] Patil V. D. and Chopde P.R. (1982) Maharashtra Univ. J., 10 (2), 157-159.

[22] Hanson W.D. (1963) Washingion, punl, 982, 125-140.

[23] Kumar S. and Chauhan B.S.P. (1982) Indian J. Agric. Sci., 52 (11), 723 727.

[24] Yadav R.K. and Gupta R.R. (1999) Crop Res., 18(1), 57-59.

[25] Kumar S., Kumar R., Kumar S., Singh S., Kumar S. and Singh M.P. (2014) International Journal of Agricultural Sciences, 10(1),356-359.

[26] Rafiq A., Danish Ibrar Mirza, Talat Mahmood M.Y., Khan M.A., Iqbal M.S and Munir Ahmad (2014) Journal of Agricultural Research (Lahore), 52(1), 43-52. 